\title{
Long QT and Cardiac Arrest After Symptomatic Improvement of Pulmonary Edema
}

\author{
MAJ James I. Gragg, DO; CPT James D. Jones, MD; and LTC Joel A. Miller, MD
}

A case of extreme QT prolongation induced following symptomatic resolution of acute pulmonary edema is both relatively unknown and poorly understood.

A bnormalities in the T-wave morphology of an electrocardiogram (ECG) are classically attributed to ischemic cardiac disease. However, these changes can be seen in a variety of other etiologies, including noncardiac pathology, which should be considered whenever reviewing an ECG: central nervous system disease, including stroke and subarachnoid hemorrhage; hypothermia; pulmonary disease, such as pulmonary embolism or chronic obstructive pulmonary disease; myopericarditis; drug effects; and electrolyte abnormalities.

Prolongation of the QT interval, on the other hand, can be precipitated by medications, metabolic derangements, or genetic phenotypes. The QT interval is measured from the beginning of the QRS complex to the termination of the $T$ wave and represents the total time for ventricular depolarization and repolarization. The QT interval must be corrected based on the patient's heart rate, known as the QTc. As the QTc interval lengthens, there is increased risk of R-on-T phenomena, which may result in Torsades de Pointes (TdP). Typical features of TdP include an antecedent prolonged QTc, cyclic polymorphic ventricular tachycardia on the surface ECG, and either a short-lived spontaneously terminating course or degeneration into ventricular fibrillation (VF) and sudden cardiac death. ${ }^{1}$ These dysrhythmias become more likely as the QTc interval exceeds 500 msec. $^{2}$

The combination of new-onset global Twave inversions with prolongation of the QT interval has been reported in only a few limited conditions. Some known causes of these QT T changes include cardiac ischemia, status epilepticus, pheochromocytoma, and acute cocaine intoxication. ${ }^{3}$ One uncommon and rarely reported cause of extreme QT prolongation and T-wave inversion is acute pulmonary edema. The ECG findings are not present on initial patient presen- tation; rather the dynamic changes occur after resolution of the pulmonary symptoms. Despite significant ECG changes, all prior reported cases describe ECG normalization without significant morbidity. ${ }^{4,5}$ We report a case of extreme QT prolongation following acute pulmonary edema that resulted in cardiac arrest secondary to VF.

\section{CASE PRESENTATION}

A 72-year-old male with medical history of combined systolic and diastolic heart failure, ischemic cardiomyopathy, coronary artery disease, cerebral vascular accident, hypertension, hyperlipidemia, type 2 diabetes mellitus, and tobacco dependence presented to the emergency department (ED) by emergency medical services after awaking with acute onset of dyspnea and diaphoresis. On arrival at the ED, the patient was noted to be in respiratory distress (ie, unable to speak single words) and was extremely diaphoretic. His initial vital signs included blood pressure, $186 / 113 \mathrm{~mm} \mathrm{Hg}$, heart rate, 104 beats per minute, respiratory rate, 40 breaths per minute, and temperature, $36.4{ }^{\circ} \mathrm{C}$. The patient was quickly placed on bilevel positive airway pressure and given sublingual nitroglycerin followed by transdermal nitroglycerin with a single dose of $40 \mathrm{mg}$ IV furosemide, which improved his respiratory status. A chest $\mathrm{X}$-ray was consistent with pulmonary edema, and his brain natriuretic peptide was $1654 \mathrm{pg} / \mathrm{mL}$. An ECG demonstrated new T-wave inversions, and his troponin increased from 0.04 to $0.24 \mathrm{ng} / \mathrm{mL}$ during his ED stay (Figure 1). He was started on a heparin infusion and admitted to the hospital for hypertensive emergency with presumed acute decompensated heart failure and non-ST-elevated myocardial infarction.

Throughout the patient's first night, the troponin level started to down-trend after peaking at $0.24 \mathrm{ng} / \mathrm{mL}$, and his oxygen requirements decreased allowing transition to nasal cannula. However, his repeat ECGs demonstrated
Author affiliations can be found at the end of the article. Correspondence: James Gragg (gragg.james@gmail.com)

Fed Pract. 2021;38(suppl 4). Published online November 15. doi: $10.12788 /$ fp. 0187 
FIGURE 1 Electrocardiogram on Presentation

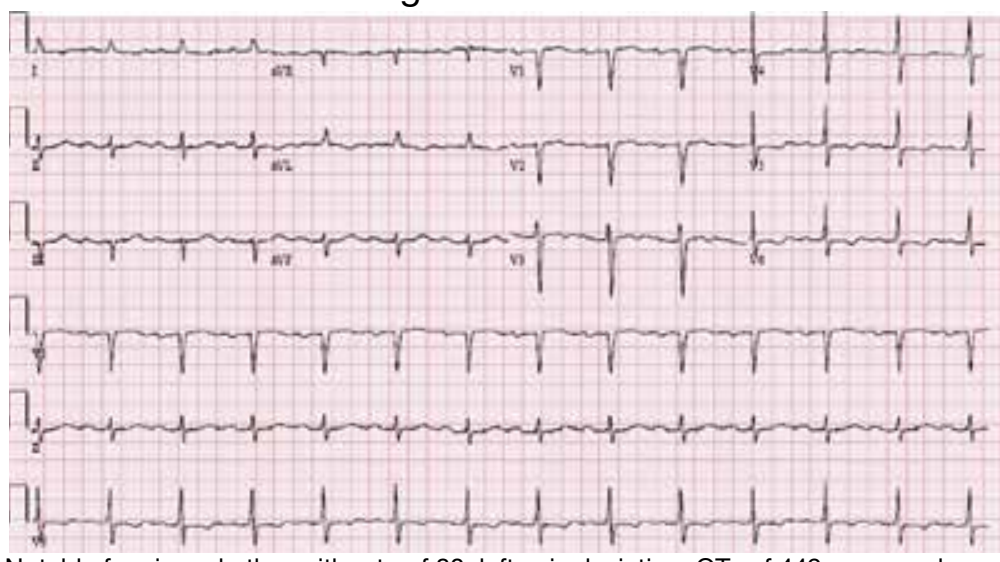

Notable for sinus rhythm with rate of 80 , left axis deviation, QTc of $449 \mathrm{msec}$ and T-wave inversion I, aVL, V4-V6.

\section{FIGURE 2 Electrocardiogram 22 Hours After Presentation}

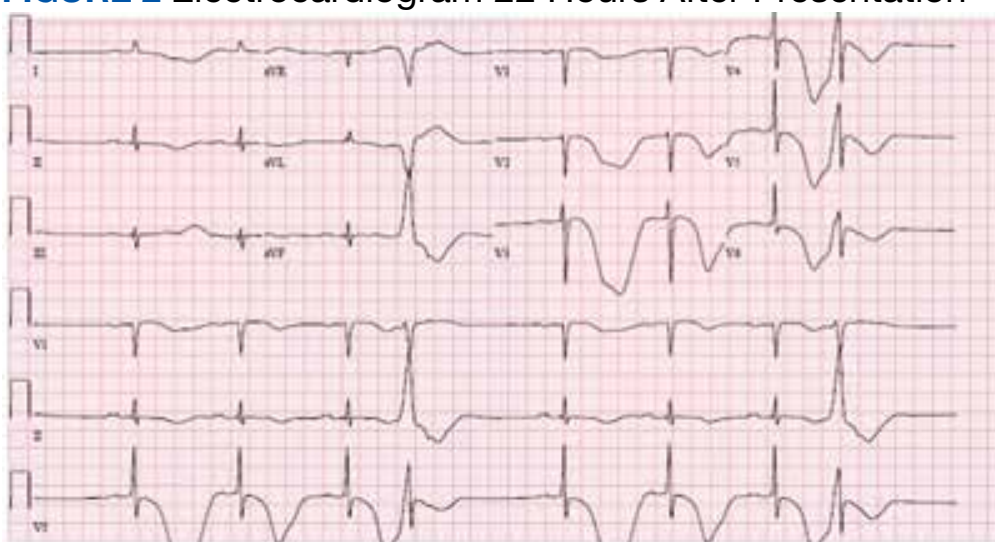

Notable for sinus bradycardia with a rate of 55 , frequent premature ventricular contraction, QTc of $703 \mathrm{msec}$ and bizarre T-wave inversions (V2-V6). Note the location of the premature ventricular contractions relative to the T waves. The patient had a ventricular fibrillation cardiac arrest shortly after this electrocardiogram was performed.

significant T-wave abnormalities, new premature ventricular contractions, bradycardia, and a prolonging QTc interval to $703 \mathrm{msec}$ (Figure 2). At this time, the patient's electrolytes were normal, specifically a potassium level of $4.4 \mathrm{mEq} / \mathrm{L}$, calcium $8.8 \mathrm{mg} / \mathrm{dL}$, magnesium $2.0 \mathrm{mg} / \mathrm{dL}$, and phosphorus $2.6 \mathrm{mg} / \mathrm{dL}$. Given the worsening ECG changes, a computed tomography scan of his head was ordered to rule out intracranial pathology. While in the scanner, the patient went into pulseless VF, prompting defibrillation with $200 \mathrm{~J}$. In addition, he was given $75 \mathrm{mg}$ IV lidocaine, $2 \mathrm{~g}$ IV magnesium, and 1 ampule of both calcium chloride and sodium bicarbonate. With treatment, he had return of spontaneous circulation and was taken promptly to cardiac catheterization. The catheterization showed no significant obstructive coronary artery disease, and no interventions were performed. The patient was transferred to the cardiac intensive care unit for continued care.

During his course in the intensive care unit, the patient's potassium and magnesium levels were maintained at high-normal levels. The patient was started on a dobutamine infusion to increase his heart rate and attempt to decrease his QTC. The patient also underwent cardiac magnetic resonance imaging (MRI) to evaluate for possible myocarditis, which showed no evidence of acute inflammation. Echocardiogram demonstrated an ejection fraction of $40 \%$ and global hypokinesis but no specific regional abnormalities and no change from prior echocardiogram performed 1 year earlier. Over the course of 3 days, his ECG normalized and his QTC shortened to $477 \mathrm{msec}$. Genetic testing was performed and did not reveal any mutations associated with long QT syndrome. Ultimately, an automated internal cardiac defibrillator (AICD) was placed, and the patient was discharged home.

Over the 2 years since his initial event, the patient has not experienced recurrent VF and his AICD has not fired. The patient continues to have ED presentations for heart-failure symptoms, though he has been stable from an electrophysiologic standpoint and his QTC remains less than 500 msec.

\section{DISCUSSION}

Prolongation of the QT interval as a result of deep, global T-wave inversions after resolution of acute pulmonary edema has been minimally reported. .,5 $^{\text {This }}$ phenomenon has been described in the cardiology literature but has not been discussed in the emergency medicine literature and bears consideration in this case. ${ }^{4,5}$ As noted, an extensive evaluation did not reveal another cause of QTc prolongation. The patient had normal electrolytes and temperature, his neurologic examination and computed tomography were not remarkable. The patient had no obstructive coronary artery disease on catheterization, no evidence of acute myocarditis on cardiac MRI, no prescribed medications associated with QT prolongation, and no evidence of genetic mutations associated with QT prolongation on testing. The minimal troponin elevation was felt to represent a type II myocardial infarction related to ischemia due to supply-demand mismatch rather than acute plaque rupture. 
Littmann published a case series of 9 cases of delayed onset T-wave inversion and extreme QTC prolongation in the 24 to 48 hours following treatment and symptomatic improvement in acute pulmonary edema. ${ }^{4}$ In each of his patients, an ischemic cardiac insult was ruled out as the etiology of the pulmonary edema by laboratory assessment, echocardiography, and left heart catheterization. All of the patients in this case series recovered without incident and with normalization of the QTC interval. ${ }^{4}$ Similarly, in our patient, significant QT T changes occurred approximately 22 hours after presentation and with resolution of symptoms of pulmonary edema. Pascale and colleagues also published a series of 3 patients developing similar ECG patterns following a hypertensive crisis with resolution of ECG findings and without any morbidity. ${ }^{5}$ In contrast, our patient experienced significant morbidity secondary to the extreme QTc prolongation.

\section{CONCLUSIONS}

We believe this is the first reported case of excessive prolongation of the QTc with VF arrest secondary to resolution of acute pulmonary edema. The pattern observed in our patient follows the patterns outlined in the previous case series-patients present with acute pulmonary edema and hypertensive crisis but develop significant ECG abnormalities about 24 hours after the resolution of the high catecholamine state. Our patient did have a history of prior cardiac insult, given the QTc changes developed acutely, with frequent premature ventricular contractions, and the cardiac arrest occurred at maximal QTc prolongation, yet after resolution of the high catecholamine state, the treatment team felt there was likely an uncaptured and short-lived episode of TdP that degenerated into VF. This theory is fur- ther supported by the lack of recurrent VF episodes, confirmed by AICD interrogation, after normalization of the QTC in our patient.

\section{Author affiliations}

James Gragg is an Active Duty Army Staff Physician, and Joel Miller is a Staff Physician at Carl R. Darnall Army Medical Center in Fort Hood, Texas. James Jones is an Active Duty Army Staff Physician at Martin Army Community Hospital in Fort Benning, Georgia. James Gragg and Joel Miller are Assistant Professors of Military and Emergency Medicine at the Uniformed Services University of the Health Sciences in Bethesda, Maryland. Joel Miller is a Reservist serving as Assistant Deputy Commander for Clinical Services for the 228th Combat Support Hospital at Fort Sam Houston in San Antonio, Texas.

\section{Author disclosures}

The authors report no actual or potential conflicts of interest with regard to this article.

\section{Disclaimer}

The opinions expressed herein are those of the authors and do not necessarily reflect those of Federal Practitioner, Frontline Medical Communications Inc., the US Government, or any of its agencies. This article may discuss unlabeled or investigational use of certain drugs. Please review the complete prescribing information for specific drugs or drug combinations-including indications, contraindications, warnings, and adverse effectsbefore administering pharmacologic therapy to patients.

\section{References}

1. Passman R, Kadish A. Polymorphic ventricular tachycardia, long Q-T syndrome, and torsades de pointes. Med Clin North Am. 2001;85(2):321-341. doi:10.1016/s0025-7125(05)70318-7

2. Kallergis EM, Goudis CA, Simantirakis EN, Kochiadakis GE, Vardas PE. Mechanisms, risk factors, and management of acquired long QT syndrome: a comprehensive review. ScientificWorldJournal. 2012;2012:212178. doi:10.1100/2012/212178

3. Miller MA, Elmariah S, Fischer A. Giant T-wave inversions and extreme QT prolongation. Circ Arrhythm Electrophysiol. 2009;2(6):e42-e43. doi:10.1161/CIRCEP.108.825729

4. Littmann L. Large T wave inversion and QT prolongation associated with pulmonary edema: a report of nine cases. J Am Coll Cardiol. 1999;34(4):1106-1110. doi:10.1016/s0735-1097(99)00311-3

5. Pascale P, Quartenoud B, Stauffer JC. Isolated large inverted $T$ wave in pulmonary edema due to hypertensive crisis: a novel electrocardiographic phenomenon mimicking ischemia?. Clin Res Cardiol. 2007;96(5):288-294. doi:10.1007/s00392-007-0504-1 